\title{
TRAS EL SILENCIO DE LA VERGÜENZA: TRAUMA, GÉNERO Y NARRATIVA CONTEMPORÁNEA
}

\author{
Maite Escudero Alías, Universidad de Zaragoza ${ }^{1}$ \\ Email: mescuder@unizar.es
}

\begin{abstract}
Resumen: El presente artículo traza una genealogía del trauma e insiste en el componente transformador y autotélico de los afectos como una forma de superación de traumas insidiosos relacionados con la formación de identidades minoritarias . Así, partiendo de marcos teóricos interdisciplinares como son los Estudios de Trauma y la Teoría de los Afectos, se explora el afecto de la vergüenza y se ofrece un paradigma epistemológico de sentir y leer literatura mediante una reparación psíquica y afectiva . Por ello, tras un exhaustivo recorrido teórico, ilustraremos de forma más pragmática cómo se llevan a cabo estas transformaciones en las novelas de Shani Mootoo en Cereus Blooms at Night (1996) y de Emma Donoghue en Room (2010) .

Palabras clave: vergüenza, trauma, afectos, género, reparación .

Título en inglés: "On the Silence of Shame: Trauma, Gender and Contemporary Narrative in English"

Abstract: Drawing on interdisciplinary theoretical frameworks like trauma studies and affect theory, the present article traces back the genealogy of trauma and highlights the self-rewarding characteristics of the affect system as one possible way to surpass the pernicious effects of insidious traumas often attached to the formation of minority identities. For this purpose, I explore shame as an affect that involves both feelings of worthlessness and a productive enactment of transformation and hope. After a thorough account of such theoretical frames, the article succinctly illustrates how this reparative discourse can indeed forge new sites of identity formation in novels like Cereus Blooms at Night (1996) by Shani Mootoo and Room, by Emma Donoghue (2010), thus challenging mainstream discourses of gender, race and affective normalcy . Keywords: shame, trauma, affects, gender, reparation .
\end{abstract}

Susan Sontag, conocida escritora y crítica literaria, nos anima a imaginar la literatura como una utopía, un lugar en el que imperan los modelos más encumbrados, casi inaccesibles . En sus propias palabras, "la literatura es, en primer lugar, una de las maneras fundamentales de nutrir la conciencia . Desempeña una función esencial en la creación de la vida interior, y en la ampliación y ahondamiento de nuestras simpatías y nuestras sensibilidades hacia otros seres humanos y el lenguaje" (2003:1) . La literatura como

${ }^{1}$ Date of reception: 19 September 2014

Date of acceptance: 6 October 2014 
herramienta de conocimiento ejerce un rol esencial no solo para conocer al Otro sino también para conocernos a nosotros mismos y ahondar en nuestros afectos para con los demás y poder desarrollar, así, cualidades como la empatía y la imaginación . De forma similar, Hannah Arendt nos recuerda que si bien la literatura nos hace un poco más libres, es la falta de imaginación la que ha llevado al ser humano a cometer las atrocidades más aberrantes a lo largo de la historia (1969). Es decir, la ausencia de reflexión y diálogo con el Otro así como la incapacidad de imaginar otras posibilidades de vivir y de sentir, han mermado el reconocimiento de otras voces en nuestra cultura . Además de esta función epistemológica de la literatura, es interesante subrayar su potencial empático y terapéutico para representar determinados traumas colectivos e individuales que impiden el desarrollo de valores esenciales como la dignidad e integridad humana . Se propone, a continuación, una forma de entender la literatura como un elemento reparador, generador de empatía y generosidad hacia el Otro, a la par que se revisan algunas nociones que conforman la tradición de los Estudios de Trauma, como pueden ser los conceptos de duelo y melancolía, con el fin de hallar nuevas vías hermenéuticas de efectiva aplicación en nuestra sociedad contemporánea .

A partir de estas premisas, el presente artículo pretende ampliar el marco teórico de los denominados Estudios de Trauma que, mayoritariamente, se ocupan de examinar la representación de diferentes traumas a través de la literatura, dotándolos con frecuencia de un sentido patológico y determinante . Esto es debido al componente paradójico que define el concepto de trauma; a saber, una suerte de aporía lingüística y epistemológica que fractura y quiebra la experiencia del tiempo de la persona que lo padece y que, por lo tanto, es difícil de superar (Caruth, 1995) ..$^{2}$ Aunque mucho se ha escrito y publicado dentro de los Estudios de Trauma, existen todavía unas líneas de trabajo no tan conocidas y que tienen que ver con la influencia del afecto de la verguienza en la construcción de la identidad . La vergüenza, como uno de los afectos constitutivos del ser humano, puede resultar decisiva para esconder o velar traumas de diversa índole . Por ello, después de experimentar un hecho traumático, bien en un tiempo presente o bien en un pasado más lejano, la vergüenza puede aparecer y perseguir a la/s víctima/s de forma incesante, causando daños psicológicos demoledores para la persona que la sufre. La vergüenza, a menudo, se define como un afecto ambivalente: necesita exhibirse públicamente por medio de signos faciales visibles (i .e . ruborizarse o bajar la mirada) aunque sea precisamente su propia exhibición lo que más repudie; interpela a la vez que requiere una audiencia, aunque también puede mantenerse en secreto y escondida. En general, es este componente paradójico el que podría definir la vergüenza como una aporía, por utilizar la terminología de Caruth (1995), una de las teóricas más relevantes dentro de los Estudios de Trauma en la literatura y cultura . Concretamente, me interesa analizar la noción de vergüenza porque, aún siendo uno de los pilares invisibles que subyacen en muchos de los traumas de carácter racial y sexual, entraña una difícil detección . Así, este artículo parte de la convicción de

\footnotetext{
${ }^{2}$ Cathy Caruth subraya el concepto de "belatedness" para sugerir que un hecho traumático solo se puede conocer después de que ha ocurrido, a través de la analepsis: "the impact of the traumatic event lies precisely in its belatedness, in its refusal to be simply educated, in its insistent appearance outside the boundaries of any single place or time" (1995:9).
} 
que es necesario detectar diferentes formas de violencia ejercidas sobre las mujeres y otros colectivos minoritarios para poder así visibilizarlas, transformarlas o erradicarlas . Para ello, resulta imprescindible complementar el marco teórico de los Estudios de Trauma con la Teoría de los Afectos, con el fin de argumentar cómo la literatura nos ofrece interesantes herramientas de detección, análisis y superación de este afecto, en principio negativo, y que sufren mayoritariamente los grupos más vulnerables, sobre todo las mujeres y otras personas que pertenecen a minorías raciales y sexuales .

\section{ESTUDIOS DE TRAUMA Y GÉNERO}

Una de las características fundamentales de los Estudios de Trauma es su notoria interdisciplinaridad. Si bien comenzó a ser estudiado a principios del siglo XX por disciplinas como la psiquiatría y el psicoanálisis, desde hace unas décadas, en concreto desde mediados de los años 90, su investigación también se está llevando a cabo desde la rama de las Humanidades, especialmente dentro de los campos de la Literatura y la Historia . Así, los Estudios de Trauma se han consolidado como importantes herramientas teóricas y analíticas, necesarias para explorar, fundamentalmente, traumas históricos como guerras, el Holocausto, atentados terroristas, etc. Fue Freud quien a lo largo de su carrera intentó definir el concepto de trauma como un desorden psíquico de la memoria. Al final de la I Guerra Mundial se refirió a la "neurosis traumática" (1918) para describir algunos de los síntomas padecidos por los soldados de guerra y fue en Beyond the Pleasure Principle (1920) cuando acunó el término de "compulsión de repetición" para aludir a la repetición compulsiva del momento traumático que a menudo aparece en forma de pesadillas. Se esperaba que, al despertar, los pacientes procesaran el dolor de forma tardía para encontrar nuevas formas de disipar el trauma retroactivamente. Después de la I Guerra Mundial aparecieron nuevas corrientes psicoterapeúticas que ayudaron a combatir distintos síntomas traumáticos hallados en los soldados que combatieron en la guerra . Es a partir de este momento cuando se acuña el término de "trauma de guerra" (shell shock), asociado, en su origen, a un tipo de histeria masculina traumática . Según el DSM IV (Diagnostic and Statistical Manual of Mental Health Disorders), los síntomas característicos de este tipo de trauma incluyen la reexperimentación del acontecimiento traumático, ya sea a través de ensoñación diurna o en forma de pesadillas . También pueden producirse estados disociativos que pueden durar desde pocos minutos hasta días . La sintomatología suele acompañarse de embotamiento psíquico o anestesia afectiva y activación del sistema nervioso autónomo mediante la hiperactividad, irritabilidad y alteraciones del sueño (DSM IV 1994: 886) .

Este trauma de guerra seguirá vigente durante la II Guerra Mundial, de modo que el modelo del soldado traumatizado por una guerra (shell shocked soldier) se convertirá en el icono institucional de las víctimas de trauma durante prácticamente todo el siglo XX . A partir de esta guerra surge la figura del veterano de guerra del Vietman como "the quintaessential stressed-out American" (Luckhurst 2008: 59), alcanzando una repercusión médica, social y cultural sin precedentes. Podríamos afirmar que esta figura inaugura una forma nueva de entender el trauma, pues comienza su impulso mediático con la llegada en 
1980 del término PTSD (Post-Traumatic Stress Disorder), definido como una experiencia que supone "actual or threatened death or serious injury, or a physical threat to the integrity of the self" (Luckhurst, 2008: 64) . Aunque la aparición del PTSD fue producto de una larga lista de neurosis padecidas por los soldados combatientes en diferentes guerras, el término también incluyó a otras víctimas de carácter colectivo, como los que sobrevivieron a los bombardeos de Hiroshima, las víctimas del Holocausto Nazi, o al pueblo Afroamericano como consecuencia de años de esclavitud . Por otro lado, este término representaba la barrera epistemológica que delimitó la legitimidad y visibilidad de ciertas víctimas sobre otras, pues si ciertos traumas (colectivos o individuales) no se hallaban recogidos bajo este epígrafe, simplemente no existían . Tradicionalmente la historia y la cultura no han reconocido ciertos traumas individuales que sufren especialmente mujeres, niños y otras minorías: nos referimos a traumas que emergen de actos violentos como violaciones, abusos sexuales a menores, racismo u homofobia . Si las instituciones médicas, legales, políticas y sociales niegan estos actos como posibles catalizadores de traumas y los desvinculan de experiencias que suponen un grave daño físico o algún otro tipo de amenaza a la integridad física o psicológica difícilmente serán tratados como traumas. Hasta que no comenzaron los movimientos de políticas de identidad y determinados estudios feministas reivindicaron su inclusión, los traumas relacionados con la violencia sexual sobre las mujeres, como las violaciones y el incesto, no fueron reconocidos como tales. Por ejemplo, aunque el denominado "síndrome de trauma por violación" fue acuñado en 1974, no obtuvo reconocimiento oficial hasta pasados 20 años, ya que hasta 1994 no fue integrado dentro del Manual Diagnóstico y Estadístico de los Trastornos Mentales ni formó parte del PTSD . Así, la imposición del PTSD, si bien contribuyó a clasificar y diagnosticar enfermedades mentales, también fue una forma de silenciar otros traumas personales menos reconocidos por las instituciones médicas y legales .

Estas premisas establecieron la base esencial de los trabajos llevados a cabo por la Escuela de Yale, entre cuyos miembros fundadores destacan Cathy Caruth, Shoshana Felman, Dori Laub, Geoffrey Hartman o Dominick LaCapra, y quienes siguiendo la lógica del pensamiento postmoderno, fueron pioneros en poner de manifiesto la irrepresentabilidad de la realidad traumática, precisamente porque el trauma desafía y escapa a los confines epistemológicos del lenguaje . En este sentido, me gustaría insistir en el rol primordial de la literatura porque sirve como vehículo de transmisión y de representación de diferentes traumas a través de técnicas narrativas y estilísticas que solo son propias de la ficción, como puede ser el uso de flash-backs o interrupciones temporales entre pasado, presente y futuro, la fragmentación, los silencios, la indirección retórica (expresar de forma indirecta o a través de metáforas el dolor indescriptible de un trauma), la disociación (hablar en tercera persona de una misma), la repetición, etc ., características todas ellas que se asemejan a los síntomas del trauma. De forma más concreta, Caruth se centra en cómo el concepto Freudiano de "belatedness" permite a la persona traumatizada sobrevivir al trauma encontrando nuevas formas de ser testigo: "the history of trauma, in its inherent belatedness, can only take place through the listening of the another" (1995: 11). Es precisamente este llamamiento y contacto con el Otro lo que lleva a Caruth a afirmar que el estudio del trauma supone una contribución importantísima para pensar 
sobre la literatura, la política o la ética . La importancia de considerar al Otro para comprender una experiencia traumática fue uno de los aspectos centrales del estudio de Felman y Laub (1992) en el que se destaca el concepto de "testimonio" como esencial en el devenir de la historia y sus traumas . Entendido como el acto de ser testigo, para estos autores la noción de testimonio permite recuperar un pasado traumático y recordarlo porque, aunque fragmentado y desconocido, continúa influyendo en la configuración del presente y del futuro. Asimismo, Felman y Laub defienden que los textos literarios sirven para enfrentarse a determinados actos horribles porque convierten a los lectores en testigos, estableciendo conexiones temporales y espaciales productivas . Esta dependencia con el Otro está relacionada con el concepto de la "ética de la alteridad" del filósofo Emmanuel Lévinas (1998), en tanto en cuanto el habla del testigo trasciende a éste porque conforma el medio a través del cual el testigo puede realizar el testimonio . Así, el testigo, desde su posición única, es un vehículo de transmisión de esta realidad traumática . Puesto que ni el hablante ni el que escucha poseen la verdad absoluta, debemos prestar atención al lenguaje de transmisión de esos traumas porque, según estos autores, "testimony is, in other words, a discursive practice, as opposed to pure theory" (1992: 5) . Como práctica discursiva, la noción de testimonio nos enseña a ser receptivos con las voces y experiencias de otros que han padecido determinados traumas y nos invita a considerar las voces silenciadas de un pasado traumático para evitar que el futuro esté determinado y enredado por repeticiones traumáticas desconocidas y no reconocidas .

Dominick LaCapra defiende que las obras de ficción pueden dotar a un fenómeno histórico traumático de nuevas perspectivas, a la vez que señala que "truth is not divorced from fiction" (2001: 13). Interesado en desmantelar oposiciones binarias del tipo verdad/ ficción o representación/irrepresentabilidad, LaCapra se apoya en conceptos psicoanalíticos como acting out/working through para adaptarlos y conectarlos con el análisis histórico, sociocultural y la crítica política . Concretamente me interesa resaltar aquí la gran utilidad de su posición dialéctica entre sujeto y objeto, diferenciando entre la objetividad del escritor o historiador y la objetualización de la víctima, aludiendo al término de empatía como factor clave para entender y responder afectivamente al trauma del Otro sin apropiarse de él. Así, nos recuerda que:

Being responsive to the traumatic experience of others, notably victims, implies not the appropriation of their experience but what I would call empathic unsettlement, which should have stylistic effects or, more broadly, effects in writing which cannot be reduced to formulas or rules of method. (2001: 41)

Esta posición de "desconcierto empático" requiere que se tenga en cuenta el papel de la experiencia y de los afectos para articular y rearticular la representación del trauma de formas que pueden - o no - trascenderlo pero que requieren un cierto dinamismo interpretativo con el fin de evitar un anquilosamiento cognitivo. Nos encontramos, por lo tanto, ante la distinción entre la noción de "acting out", entendida como una repetición compulsiva de la representación del trauma y de "working through", que se refiere a la superación de los síntomas traumáticos o, al menos, al éxito en conseguir una cierta mitigación de los efectos del trauma . Para LaCapra, la noción de "working through" no se 
ha teorizado demasiado, pues existe una cierta fijación entre la mayoría de los críticos del trauma en la repetición compulsiva del "acting out" con fragmentaciones ilimitadas, aporías y mecanismo restrictivos que no hacen sino reforzar el fantasma de discursos totalitarios . El problema radica en que estos discursos buscan ante todo la expresión de una dinámica maniquea del trauma, donde la víctima o bien lo supera o por el contrario lo representa una y otra vez . La crítica que realiza LaCapra a esta dualidad permite encontrar posiciones intermedias que eviten narraciones redentoras donde el trauma se niega y repeticiones sublimes donde el trauma se magnifica. Más bien, como trata de reflejar el presente estudio, comparto con este autor la creencia de que es posible moverse más allá de la citada dicotomía a través de la resignificación de los afectos involucrados en un determinado trauma.

La década de los 90 fue también decisiva para la propagación de estudios sobre el trauma de la identidad que buscaban la visibilidad de los traumas sexuales sufridos por mujeres y por otros colectivos minoritarios como pueden ser las lesbianas, gays, personas transgénero y transexuales. Estos progresos se deben en parte a una serie de trabajos pioneros que no han gozado de reconocimiento académico pero que fueron decisivos para la visibilidad y superación de estos traumas individuales y que me gustaría mencionar de forma sucinta . Obras como Against Our Will: Men, Women and Rape publicada por Susan Browmiller en 1975 nos alerta del vacío legal, social y académico que ha sufrido el término de violación. Fundamental en su trabajo es la idea de que la violación tiene una historia y que como tal es necesario contarla discursivamente . En 1986 Diana Russel publicó The Secret Trauma: Incest in the Lives of Girls and Women, un estudio sobre el incesto y la necesidad de reconocer legalmente a las víctimas de incesto como víctimas de un trauma violento con el fin de prestarles la ayuda necesaria . La aparición de libros de auto-ayuda también fue decisiva para recuperar la memoria de estas víctimas ya que muchas de ellas padecen amnesia por los abusos sufridos durante su niñez . Bajo el optimista título de "healing is possible", muchos de estos libros enfocan por primera vez el abuso sexual como un trauma que se puede superar .

Judith Herman, conocida psiquiatra feminista, subraya en su obra Trauma and Recovery: The Aftermath of Violence - From Domestic to Political Terror (1992), la importancia de recordar y contar la verdad sobre estos actos violentos cometidos sobre las mujeres con el fin de no patologizar a la víctima, a menudo encasillada y definida como una persona masoquista y más vulnerable a sufrir determinados abusos . ${ }^{3}$ Para esta autora es urgente desenmascarar al verdugo y no silenciar sus crímenes, y para ello hace un llamamiento a la colaboración institucional, fundamentalmente la médico-legal . Siguiendo esta línea revisionista del concepto de trauma destaca la obra de Kalí Tal, Worlds of Hurt: Reading the Literatures of Trauma (1995), donde la autora redunda en la idea de que es a través de la literatura y la cultura que la noción del trauma psíquico puede

\footnotetext{
${ }^{3}$ A mediados de los 80 varios estudios psicológicos propusieron introducir el término "desorden de personalidad masoquista" en el Manual Diagnóstico y Estadístico de los Trastornos Mentales III para definir el comportamiento de determinadas mujeres que permanecen en relaciones en las que otros las explotan y abusan de ellas a pesar de tener oportunidades para cambiar estas situaciones . Este posicionamiento no hace sino reforzar los discursos patriarcales que niegan, una vez más, la agencia de estas víctimas .
} 
ser ampliada y revisada políticamente . Por ello, denuncia el conservadurismo en el tratamiento de los traumas reconocidos oficialmente, y denuncia la tendencia patriarcal de culpar a las mujeres, desplazando así la responsabilidad y culpa de los hombres sobre las víctimas indefensas. Asimismo, es importante su reflexión sobre el concepto de testimonio ya que para Tal el testimonio solo es eficaz si existe un testigo empático dispuesto a prevenir la repetición del trauma sexual, algo que a veces el status quo no está dispuesto a realizar. Así, el testigo se convierte en un vehículo de transmisión de esa realidad traumática .

Otro de los estudios más destacados que se centran en los testimonios de víctimas de incesto y violencia sexual expresados a través de la literatura es el trabajo de Suzette Henke, Shattered Subjects: Trauma and Testimony in Women's Life-Writing (1998) . Aquí, Henke insiste en la importancia del testimonio literario autobiográfico como un modelo constructivo de contar, interpretar y transformar el trauma . Lo más novedoso de su estudio es el acuñamiento del término "escrito-terapia", que se convertirá en uno de los paradigmas feministas de los estudios de trauma, además de infiltrarse en los colectivos de terapeutas, psiquiatras o narratólogos . Con esta noción, Henke argumenta que relatar un trauma sexual en primera persona constituye un método terapeútico de recuperar la agencia y autoridad para muchas mujeres . El esfuerzo de autoría ofrece el potencial de curación o alivio de síntomas persistentes como la apatía, disforia o flashbacks incontrolables. Para desarrollar el concepto de escrito-terapia es preciso generar nuevos modos de representación literaria o incluso nuevos géneros narrativos con los que examinar el impacto de experiencias traumáticas y cómo superar la fragmentación psíquica a través de las mismas . Por ello, es partidaria de ampliar el género de la autobiografía y habla de la inclusión de otros como el género epistolar, diarios personales o memoirs. Tanto la autobiografía, como forma poderosa de escrito-terapia que ofrece la posibilidad de reinventar el yo y reconstruir el sujeto femenino traumatizado e invisibilizado por la historia y el lenguaje, como estos nuevos géneros, en tanto en cuanto permiten a la narradora reinterpretar su pasado y los códigos sociales e ideológicos que la definen, se convierten en discursos testimoniales que dotan de una agencia discursiva - y por ende ontológica - a mujeres traumatizadas, ya que, quizá, de otra manera, nunca obtendrían el reconocimiento o legitimidad como supervivientes de un trauma sexual .

Conviene señalar que todas estas obras inciden en la importancia de lograr que los traumas insidiosos sean reconocidos y visibles como tales: con el adjetivo insidioso me refiero a experiencias de un sexismo muy sutil al que se exponen las mujeres a diario y que, añadidas a traumas como la violación, forjan conexiones entre el trauma y otras formas de opresión que están normalizadas socialmente. En este mismo sentido se expresa Ann Cvetkovick, reconocida estudiosa del concepto de trauma y sexualidad, en su libro An Archive of Feelings: Trauma, Sexuality and Lesbian Public Cultures (2003) . Como parte de su proyecto, lo que le interesa a esta autora es ver cómo la ansiedad, la depresión, la ira o la vergüenza por pertenecer a un colectivo minoritario tienen el potencial de ser transformadas. Es más, para convertir estas formas de violencia insidiosa en prácticas reparadoras es necesario repensar el impacto psíquico de las víctimas y crear nuevas rutas de trabajo . 


\section{LA VERGÜENZA MELANCÓLICA COMO TIPO DE VIOLENCIA INSIDIOSA}

Mi interés en el afecto de la vergüenza obedece a un compromiso personal y académico por intentar encontrar respuesta a los efectos demoledores que puede provocar la vergüenza en la vida de ciertas personas, bien como consecuencia de violaciones y abusos sexuales o bien por otros actos violentos insidiosos como pueden ser el sexismo, el racismo o la homofobia . No por casualidad, la vergüenza es el "afecto de la indignidad, de la humillación, de la derrota y de la alienación [...] la vergüenza golpea en lo más profundo del corazón y sus heridas penetran en el interior de nuestro yo como si fueran una enfermedad del alma" (Tomkins, 1995: 133). La vergüenza es ubicua a la experiencia de todos los seres humanos y, como tal, atraviesa e impregna diferentes culturas, religiones y naciones . Habita con nosotros desde que nacemos y permanece presente en las relaciones afectivas humanas y en los procesos de comunicación . La experiencia fenomenológica de una persona con vergüenza es la de un deseo intenso de esconderse, desaparecer o morir . Entraña un estado altamente doloroso que, al igual que el trauma, puede desencadenar un comportamiento desequilibrado, confusión en el pensamiento, disolución del yo, amnesia o incluso incapacidad para hablar. A diferencia de otros afectos, como la culpa, el asco o el orgullo, la vergüenza ataca el sistema global de la autoestima de la persona que la padece y actúa como una señal de alerta ante un fracaso personal y/o social delante del otro . Es precisamente la exposición pública del fracaso del yo ante el otro lo que delata al cuerpo avergonzado, ya que éste muestra signos evidentes de auto-negación así como de una violencia del sujeto contra sí mismo .

La mayor parte de los estudios han subrayado que una de las características de la vergüenza es que siempre lleva consigo un miedo de verse expuesta a una desnudez psicológica o física que tiene como referente al otro. Freud ya señaló en su estudio sobre la neurosis infantil que la exposición de los órganos sexuales o del cuerpo desnudo, asî como los pensamientos sobre ello, evocaba sentimientos de vergüenza . Asimismo, también destacó otros sentimientos como la pérdida de control de las funciones corporales, de los impulsos, de restricciones emocionales o de desequilibrios sociales como agentes catalizadores de la vergüenza. La mayor parte de autores, desde Darwin y Freud hasta los más contemporáneos, hacen hincapié en que el deseo de esconderse y de evitar la exposición es intrínseco a la experiencia de la vergüenza (Tomkins 1995; Segdwick 2003; Ahmed 2004; Pajaczkowska y Ward 2008) . Por ello, todos coinciden en señalar una topografía corporal de este sentimiento que se manifiesta a través de signos y expresiones faciales que la delatan; a saber, bajar la cabeza, retirar o bajar la mirada, encoger el cuerpo o ruborizarse. Sin embargo, la vergüenza individual no siempre se manifiesta a través de signos corporales evidentes, y como afecto poderoso y doloroso que es, también puede alcanzar proporciones abrumadoras para la persona que la padece si se muestra de forma permanente y no visible. Por ello, diferenciaré entre lo que he acuñado como "vergüenza melancólica", que se encuentra asociada al término de melancolía dado su carácter patológico prolongado en el tiempo, y una vergüenza normativa, que aparecería de forma puntual en situaciones embarazosas o de humillación . El carácter transitorio - quizá por ello más visible - de la vergüenza normativa la convierte en un afecto constructivo que 
podría ayudar a las personas a regular su sentido del bienestar al alertarle de sus posibles efectos negativos . Por el contrario, la vergüenza melancólica requiere que ciertos espacios sociales y corporales se deformen y reformen, puesto que los cuerpos avergonzados tienden a esconderse de los que presencian su vergüenza . Esta evasión puede ser muy dolorosa y conducir a la persona a padecer experiencias muy prolongadas de vergüenza, cuya intensidad y persistencia pueden incluso llevarle al suicidio. Así, este tipo de vergüenza, precisamente porque entraña una difícil detección y una falta de reconocimiento académico, médico y social, necesita ser reconocida con el fin de paliar y curar sus efectos devastadores.

En este punto, conviene distinguir, además, entre una vergüenza nacional, propia de un colectivo o nación que ha perpetrado violencia y/o sometido a sus habitantes a humillaciones, violaciones, vejaciones, etc ., y la vergüenza individual, puesto que existen notorias diferencias . A diferencia de la vergüenza individual, la visibilidad y temporalidad de la vergüenza colectiva se lleva a cabo a través de mecanismos de poder que insisten en llorar pública y globalmente ciertos tipos de vergüenza en detrimento de otros que son permanentemente velados . Por ejemplo, la constante atención a determinados acontecimientos históricos como el Holocausto Nazi o el 11-S contrasta con la escasa repercusión social y cultural de otros genocidios como el de Armenia o el de Ruanda . De la misma manera, se crea una jerarquía de victimización, por la cual existen víctimas reconocidas frente a otras que no lo son, multiplicándose, por lo tanto, el sentimiento vergonzante de ser una víctima irreconocible e ilegítima. Si bien los efectos políticos de la vergüenza se observan con mayor facilidad en algunos países como Alemania, Estados Unidos o Australia, notoriamente más dispuestos a llorar su vergüenza nacional por haber participado en genocidios, guerras u otros conflictos violentos, también es cierto que a menudo, tras historias de ocupación y dominación violenta, se esconde una dinámica de vergüenza contagiosa porque ésta se desplaza del colectivo o grupo social al individuo en particular. De hecho, la vergüenza es una emoción que fluye rápidamente, posee una propiedad contagiosa que hace que circule y se pueda intercambiar con intensidad entre colectivos de personas tradicionalmente estigmatizadas y excluidas como pueden ser las personas pobres, los inmigrantes, las personas homosexuales o las prostitutas . Es como si el estigma de la vergüenza nacional se desplazara a otros cuerpos individuales, más susceptibles de internalizar el estigma de la vergüenza y de reproducir su patología específica.

Históricamente ha sido la disciplina del psicoanálisis la encargada de estudiar el afecto de la vergüenza y aún así, la vergüenza se ha visto marginada en aras de otros como la culpa, el asco o el narcisismo. Es a partir de la década de los 90 cuando comienza un interés creciente por estudiar este afecto desde diferentes disciplinas académicas como pueden ser desde el propio psicoanálisis, estudios de psicología, feministas, culturales y queer, subrayando así la necesidad de construir una epistemología interdisciplinar sobre la vergüenza . De esta conjunción de conocimientos parte mi interés por este afecto, sobre todo porque, a pesar de los claros paralelismos con el concepto de melancolía, ni siquiera se contempla dentro de los Estudios de Trauma . Cuando la vergüenza es persistente y patológica conduce a estados intrapsíquicos que pueden producir la disolución y la 
alienación del individuo, tanto en su vertiente social como personal . Además, la vergüenza constituye el vínculo afectivo que ha regulado y definido la construcción de las identidades minoritarias, como puede ser la identidad femenina, la racial y la homosexual . No por casualidad, la noción de orgullo, tan intrínseca a estas minorías, es facilitada por la vergüenza, al mismo tiempo que opera como un mecanismo de defensa del sujeto para protegerse de la presencia invasora de la vergüenza . Lejos de centrarme en la dicotomía vergüenza/orgullo, un campo ya muy estudiado desde los Estudios gays, lesbianas y queer, mi posicionamiento insiste en hacer visible la vergüenza - en lugar del orgullo - con la finalidad de reconsiderar su presencia y abrazarla para transformarla, en lugar de rechazarla . Asimismo, abarca la experiencia de la vergüenza como un mecanismo para pensar sobre identidades y deseos no normativos, sobre las relaciones de inclusión/exclusión social y sobre cómo este afecto puede poseer, además, un potencial transformador para la superación de ciertos traumas de índole sexual y racial. Finalmente, el énfasis en este afecto viene motivado por la escasa representación que tiene dentro de los estudios de la literatura y del trauma, tradicionalmente más ocupados en explorar otros efectos del trauma catalogados y clasificados en manuales de diagnóstico oficiales como el Manual Diagnóstico y Estadístico de los Trastornos Mentales (DSM), publicado por la Asociación Americana de Psiquiatría a lo largo del siglo XX y cuya última edición revisada data de mayo del $2013^{4}$.

En su famoso ensayo "Duelo y Melancolía" (1917), Freud escribió que el duelo habitualmente consiste en la reacción "a una pérdida de una persona querida o de algún concepto abstracto como un país, libertad o ideal, etc" (1957: 243) . Por el contrario, la melancolía resulta de la incapacidad de resolver el dolor causado por la pérdida de ese objeto, lugar o ideal. Mi argumentación parte de la definición de este ideal, que para muchas mujeres está vinculado con un sentimiento de dignidad y de agencia . Si además, estas personas proceden de otras culturas y razas y son lesbianas y queer, la pérdida se haría extensible al ideal de ser occidental, de raza caucásica y heterosexual . Esto no quiere decir que todas las mujeres, las no heterosexuales, no blancas y no occidentales padezcan vergüenza melancólica, sino más bien habría que matizar que, por estar históricamente sometidas al ridículo y a múltiples formas de rechazo y violencia como el sexismo, el racismo y la homofobia, son más vulnerables de padecerla . No obstante, como veremos a continuación en el análisis de las obras de ficción, mi hipótesis distinguirá entre los personajes que son capaces de superar la pérdida de esos ideales - y por ende su vergüenza - y aquellos que permanecen atrapados en su posición vergonzante y fracasan en resolver esas pérdidas. Cuando los personajes no son capaces de transformar su vergüenza, ésta da origen a prácticas aberrantes y violentas como pueden ser violaciones, incesto, suicidio y asesinatos . Sin embargo, hay ocasiones en las que algunos protagonistas consiguen

\footnotetext{
${ }^{4}$ Es más, la vergüenza tampoco se ha incluido en el Manual Diagnóstico y Estadístico de Trastornos Mentales como síntoma central asociado al PTSD, a diferencia de la culpa que sí aparece por su relación con los veteranos de guerra que experimentan sentimientos de culpa por haber sobrevivido a guerras o por las atrocidades que cometen . Desde el campo de la psiquiatría, autores como Budden reclaman que se incluya a la vergüenza dentro del mencionado Manual con el fin de reconocerla y tratarla como posible causa de determinados traumas insidiosos.
} 
transformarla en otros afectos a través de su desplazamiento y conversión en otros vínculos afectivos positivos .

\section{LA TEORÍA DE LOS AFECTOS Y SU POTENCIAL TRANSFORMADOR A TRAVÉS DE LA LITERATURA}

Silvan Tomkins, conocido psicólogo norteamericano, realizó el primer estudio riguroso sobre los afectos en su obra Affect Imagery Consciousness (1962-1992) . Uno de los grandes logros de Tomkins fue el de diferenciar los impulsos de los afectos, así como señalar algunas confusiones de Freud al respecto. Tomkins destacó la maleabilidad de los afectos que, a diferencia de los impulsos, pueden ser autotélicos - i.e. autosuficientes, no dependientes de recompensas externas a uno mismo, y donde sea el propio sujeto el que es capaz de redirigir el afecto de forma positiva en aras de su propio beneficio. Para Tomkins, la gran distinción entre el sistema de los afectos y el de los impulsos radica en la capacidad que tienen los primeros en generar una mayor complejidad y libertad tanto estructural como temporal . Esta cualidad permite que la relación entre el afecto y su objeto no esté constreñida a su objetivo o tiempo . Existen varios grados de libertad que dependen de la capacidad de la persona bien para sentirse fuerte o débil durante un breve espacio de tiempo o durante toda su vida . Estos grados constituyen la libertad esencial de los afectos y de las personas. Para Tomkins, los impulsos, a diferencia de los afectos, poseen una libertad restringida, basada en la urgencia biológica de satisfacer impulsos como la sed, el hambre o la acción de inspirar y expulsar aire para vivir . Además, los afectos están dotados de una mayor libertad en el tiempo, pues en teoría las personas pueden vivir sin dolor, sintiendo dolor ocasional o en un dolor constante. A diferencia de los impulsos, que tienden a incrementar su intensidad para satisfacerlos, los afectos pueden variar su intensidad, aumentándola o disminuyéndola . Así la vergüenza interna melancólica puede oscilar en el tiempo y en su intensidad y entrañar reacciones diferentes: se puede sentir vergüenza de forma muy intensa en un período muy breve de tiempo o viceversa . En ambos casos lo interesante es que la densidad e intensidad del afecto puede variar y oscilar, a diferencia de los impulsos, más dependientes de una densidad constante y regular. Son precisamente estas contingencias estructurales y temporales de los afectos las que nos permiten pensar en su transformación y cambio .

Otra característica relevante de los afectos es la libertad de la que disponen para vincularse con otros objetos y afectos distintos; en palabras del propio Tomkins, "cualquier afecto puede estar vinculado a cosas, personas, ideas, sensaciones, ambiciones, instituciones, etc . así como a otros afectos . Por lo tanto, una puede sentirse disgustada por sentir rabia o vergüenza pero también sorprendida por sentir gozo o alegría" (54). Esta libertad de los afectos para vincularse a todo tipo de objetos, personas, ideas o incluso a otros afectos es precisamente lo que los dota de un potencial que no disfrutan los instintos, mucho más limitados debido a su instrumentalidad . De esta forma, los afectos en general y la vergüenza en particular posee el potencial de ser transformada en otros afectos más positivos . No en vano, Tomkins reconoce que el afecto de la vergüenza se puede contrarrestar e interrumpir cuando pensamos, sentimos o hacemos algo placentero . Según 
este autor, la vergüenza funciona como un afecto regulador de los afectos que él denomina "Interest-Exciment" y "Enjoyment-Joy", afectos básicos conectados con nuestros deseos, esperanzas, sueños, anhelos y amores. En definitiva, éstos son afectos positivos imprescindibles para reducir los negativos y activar sentimientos de gozo para con la vida - Según Tomkins, “el gozo y la emoción proporcionan recompensas que nos permiten contrarrestar el miedo, la angustia y la vergüenza" (1995: 57). Si los afectos positivos se pueden activar por la reducción de los negativos, las personas pueden aprender a tratar el afecto de la vergüenza melancólica como un estado transitorio, contingente al cambio y a la resignificación. Según Tomkins, a pesar de la toxicidad de la vergüenza y de los estados alienantes a los que puede conducir, el afecto de la emoción, del interés o del gozo pueden desplazar la vergüenza en cualquier momento y vincularse a otros afectos positivos como el deseo o el amor .

$\mathrm{Al}$ igual que para superar ciertos traumas individuales existen mecanismos psíquicos de defensa como la represión, la supresión del afecto, desplazamiento o inhibición es posible encontrar fuentes de motivación que conviertan la vergüenza melancólica en otros modelos más positivos de vivir y de existir . Así, el período de shock cognitivo, incoherencia, confusión y aniquilación del yo puede combatirse bien a través de maniobras interpersonales como la distracción o mediante la creación de nuevos vínculos afectivos con otras personas, animales, plantas u objetos . De este modo, la transformación de la persona avergonzada en una motivada y entusiasmada por superar ese estado aniquilador se podría llevar a cabo a través de lo que aquí denomino una ética del amor o del cuidado no sólo hacia las personas, sino también hacia el mundo natural y de los objetos . La expresión "una ética del amor" evoca la idea fundamental del filósofo Emmanuel Levinas (1998) y sus propuestas para desarrollar una "ética de la alteridad" y de respeto hacia el otro, donde lo fundamental no es relacionarse momentáneamente con el otro sino ser capaces de experimentar un dinamismo que nos lleve hacia lugares y posicionamientos diferentes, que alteren positivamente cualquier suerte de visión traumática y alienante. Sin embargo, mi trabajo pretende ir un paso más allá e incluir en el concepto del "otro" a plantas, animales u objetos inanimados como parte de los vínculos constitutivos de un nuevo yo que puede renacer a partir del trauma vergonzante . Es precisamente la maleabilidad y una mayor libertad del afecto de la vergüenza las que nos permiten explorar el concepto de vergüenza melancólica y su capacidad para generar otros vínculos afectivos mucho más productivos .

Estas perspectivas transformadoras de los afectos inauguradas por Tomkins han sido retomadas en las últimas décadas dentro del feminismo y la teoría queer. Dentro del pensamiento feminista existen varias corrientes teóricas que analizan cómo los afectos superan el antropomorfismo y por lo tanto, deben considerase, además, vínculos que se crean con animales, plantas e incluso objetos inanimados (Cuomo, Munt, Ahmed) . Estos planteamientos resultan útiles porque permiten exhibir el potencial ético de formas no jerárquicas de opresión y dominación para con los afectos. Por ejemplo, Chris Cuomo defiende la articulación de una ética feminista y ecológica que esté libre de estereotipos de identidad de género y denuncia las conexiones existentes entre la misoginia, el sexismo, el racismo y la homofobia con el maltrato del mundo natural. Nos habla del concepto de 
"florecimiento" (flourishing) como un proceso ético que implica la creencia en el ser humano como agente capaz de relacionarse con el otro de forma igualitaria así como a la floreciente recuperación de un estado psíquico emocional negativo. Destaco la importancia de este concepto porque como veremos, a continuación, resulta productivo para el análisis de la vergüenza en algunas obras narrativas .

\section{NARRATIVA CONTEMPORÁNEA Y RESILIENCIA}

En este último apartado ilustro, de forma más pragmática, todo lo anteriormente expuesto, centrándome para ello en el análisis de dos obras de ficción contemporáneas escritas en lengua inglesa . En primer lugar, abarcaré sucintamente la novela Cereus Blooms at Night (1996) de Shani Mootoo, escritora de origen indo-caribeño y que reside actualmente en Canadá, y en segundo lugar me referiré a Room (2010), novela de Emma Donoughue, escritora irlandesa también residente en Canadá . En las novelas de Shani Mootoo, las mujeres están sometidas a múltiples opresiones, pues no son blancas occidentales, a veces no son heterosexuales y si lo son, están discriminadas por sufrir abandonos familiares, vejaciones y humillaciones dentro de su comunidad. Será precisamente este sentimiento de expulsión y de rechazo el que defina la génesis de una vergüenza traumática, provocada por diversas formas de violencia pues, como ya afirmara Salman Rushdie, "la vergüenza reside en la raíz de la violencia" (1983) . En nuestro caso, la vergüenza aparece como un estigma estrechamente relacionado con el grado de deshonor al que se ven expuestas las familias cuyas hijas, madres o esposas no cumplen con las obligaciones establecidas para ellas . En Cereus, Mootoo denuncia cómo la violencia extrema que se ejerce en los procesos de colonización, incesto y abusos sexuales es, asimismo, fuente de vergüenza no solo para la víctima sino también para los perpetradores . Esta novela nos permite distinguir entre una vergüenza auto-destructiva y devastadora para Chandin, el verdugo violento, avergonzado de los orígenes indios de su familia, de su piel oscura y religión ${ }^{5}$ y la que sufre su hija Mala, una vergüenza melancólica maleable, capaz de transformarse por medio de vínculos afectivos que le permiten reconectarse con la vida, si bien momentáneamente .

Por medio de la historia de su protagonista femenina, Mala Ramchandin, Cereus se convierte en un archivo de afectos que muestra los traumas insidiosos que producen el colonialismo, la violencia sexual y la racial . A través de una espiral en el tiempo de sesenta años, el narrador de la historia, Tyler, un enfermero homosexual, nos cuenta la vida de la familia Ramchandin, descendientes de trabajadores de la India que se establecieron en Lancatamara, isla ficticia situada en el Caribe, donde se desarrolla la acción. Chandin Ramchandin, de origen indio, fue adoptado por el reverendo del lugar, un misionario cuya misión es convertir a los indios a la religión cristiana . Chandin adopta las costumbres occidentales y se enamora de su hermanastra Liviana, pero debido a la ilegitimidad de esta

\footnotetext{
${ }^{5}$ Tal y como confiesa nos el narrador: "Chandin began to hate his looks, the colour of his skin, the texture of his hair, his accent, the barracks, his real parents and at times even the Reverend and his god . [...] His children's skin seemed suddenly too dark and their manner of talking crude. He wanted to remove himself from his wife and his children but knew it was impossible" (Mootoo, 1996: 33, 51).
} 
relación incestuosa, es obligado a casarse con Sarah, una mujer de su mismo origen indio, a la que repudiará y nunca amará . Chandin y Sarah tienen dos hijas, Asha y Mala . Sarah, su mujer, se enamora de Lavinia, la hermanastra de Chandin, y ambas huyen del lugar dejando involuntariamente a sus hijas en manos de Chandin . Esta huida es la escena original del trauma de las niñas, especialmente de Mala, pues su padre comienza a violarla de forma constante . Asha consigue huir, dejando a Mala doblemente abandonada . Con el paso de los años, Mala mata a su padre accidentalmente en un pelea y, rechazando habitar el espacio privado de la casa donde se han cometido todos los abusos sexuales, vivirá durante cuarenta años en el jardín, rodeada de animales y plantas, especialmente del cactus Cereus . Tyler, narrador empático por excelencia, nos cuenta la historia de Mala cuando ésta es internada ya en su vejez en un psiquiátrico y tiene que cuidar de ella .

Cuando Mala llega al psiquiátrico "Paradise Alms", está absolutamente paralizada por su miedo y la violencia de sus memorias. Tyler nos la describe como "flesh and bones with a skeletal structure, full of fear even as she slept under sedation, she was uncommunicative and made no sounds besides crying, moaning, wailing, and sighing (23)" . La memoria traumatizada es inmóvil, estática, elude el orden temporal lógico de la narración. Mala representa la condición de víctima traumatizada, pues su parálisis verbal y psíquica se incrementa por los recuerdos y memorias violentas sobre el abuso de su cuerpo . Como nos recuerda el narrador: "Her body remembers . Mala remembers" (174) . Aunque los episodios narrativos de violencia psicológica y física son estremecedores, precisamente como denuncia de estas violencias extremas, Mootoo consigue retratar al personaje de Mala con un aura de esperanza y de curación. El vínculo que se establece entre Tyler y Mala es interesante porque, aunque nunca hay ninguna comunicación verbal entre ambos - Mala no expresa nada a través de la palabra - esta narración pone de manifiesto cómo determinados traumas exceden los límites del lenguaje y la cultura .

Leer e interpretar a Cereus como una novela de reparación psíquica requiere un proceso de empatía y de vinculación con la sensibilidad extrema que emana Mala. Ya desde niña, Mala se muestra muy respetuosa con los animales y plantas que la rodean y, a diferencia de otros niños, ella sufre "cuando el tallo de una planta es cortado y separado de su flor" o cuando los niños "pisotean y matan a los caracoles" (69). Será esta sensibilidad para con el mundo natural junto con su imaginación las que salven a Mala en última instancia . La conexión y fusión de Mala con las plantas y animales del jardín, conexión por la cual es tratada como una mujer loca por los habitantes de Lancatamara, le permiten transformar su trauma en belleza y emoción hacia la naturaleza . Solo cuando Mala contempla con placer los pájaros, insectos y toca las plantas, su vergüenza melancólica encuentra una reconexión con la vida:

As night fell she dragged her rocking chair down the back stairs into the yard under the fringes of the giant mudra tree. She sat upright like a concert director in front of the wall . As the night unwound she witnessed the slow dance of huge, white cereus buds - she counted sixty- two trembling as they unfolded against the wall, a choreography of petal and sepal opening together, sending dizzying scent high and wide into the air. The moonlight reflected off the blossoms' pure whiteness and cast a glow over the yard. Mala basked (134). 
La vida de Mala es y refleja la ambivalencia del extraño cactus llamado Cereus, "sin flores, es horroroso, sin embargo, cuando florece una vez al año, es asombrosamente precioso" (22) . La dualidad de la planta es repulsiva, al igual que las violaciones de Chandin sobre Mala. Mala solo florece, momentáneamente, como la contingencia temporal del afecto de la vergüenza, como el Cereus, todo un símbolo de metamorfosis y mutabilidad. Aunque la novela nos presenta otras historias conectadas con los protagonistas, como el amor frustrado de Mala con un entomólogo llamado Ambrose Mohanty, quien la abandona a su suerte cuando conoce los abusos de su padre o del hijo transexual de Ambrose y de su enamoramiento de Tyler, lo más novedoso en la novela es que la vergüenza melancólica de Mala es productiva y no patológica, pues es capaz de generar sentimientos de regeneración y esperanza .

Siguiendo esta misma línea transformadora de los afectos negativos, nos encontramos con la novela de Emma Donoghue, Room, una historia inspirada en el caso real de Josef Fritzl, el hombre austríaco que encerró y violó a su hija durante 24 años en un bunker. Si bien semejante acto aberrante y atroz se puede prestar a creaciones fílmicas o literarias sensacionalistas y morbosas, Emma Donoghue se aleja totalmente de esta tendencia . Escrita con una delicadeza suprema y un estilo muy afectivo, esta novela representa el emblema de lo que podemos llamar narrativas de resiliencia contemporáneas . La resiliencia, concepto que proviene del campo de la física para referirse a la resistencia de los materiales en el sentido de su capacidad para recuperar su forma original después de haber sido doblados o aplastados, se define en psicología como la capacidad psíquica de las personas de resistir y redirigir un determinado trauma .

Boris Cyrulnik, neurólogo, psiquiatra y autor de numerosos libros, fue uno de los fundadores de la teoría de la resiliencia en Francia. Para Cyrulnik, la resiliencia se trata de "un proceso, de un conjunto de fenómenos armonizados en el que el sujeto se cuela en un contexto afectivo, social y cultural . La resiliencia es el arte de navegar en los torrentes . Un trauma ha trastornado al herido y le ha orientado en una dirección a la que le habría gustado no ir" (2011: 8) . En esta situación, la persona resiliente apela a sus recursos internos para resistir a las magulladuras de la herida psicológica y obedecer, en cambio, a sus impulsos de reparación psíquica . Aunque Cyrulnik habla de determinados factores que determinan la resiliencia, sin duda el papel de la representación y la narrativa es esencial . Uno de los factores de la resiliencia es, de hecho, la capacidad para organizar la propia historia, puesto que, "a partir del momento en que un niño habla, su mundo se metamorfosea" (2011: 17) . Como veremos en Room Jack debe metamorfosear su mundo como técnica de supervivencia.

Room es narrada por Jack, un niño de cinco años, nacido en cautividad como consecuencia del rapto y secuestro de su madre Ma por Old Nick, apelativo que en inglés se refiere al demonio. Jack y su madre viven en una habitación de doce metros cuadrados, donde reciben la única y temida visita de Old Nick, para llevarles comida y seguir violando a Ma cada noche . El potencial de los afectos para vincularse a cualquier objeto es fundamental para la supervivencia de Jack, puesto que la humanización que Jack realiza sobre todos y cada uno de los objetos de la habitación es un signo de sus intentos incesantes de crear vínculos afectivos con ellos, como si éstos fueran testigos empáticos. En este 
sentido, el uso de las mayúsculas para apelarlos es revelador: así se refiere a Baño, Jabón, Mesa, Puerta, Armario, Lámpara, Alfombra o Planta. Inmerso en su rutina diaria, la vida de Jack oscila entre momentos felices jugando con su madre y escuchando los cuentos formidables que ésta le cuenta, su soledad extrema cuando "Ma está ida" (Donoghue, 2010: 74) y su miedo aterrador cuando oye el sonido de la puerta que anuncia la llegada de Old Nick . Como decíamos, su supervivencia depende de los vínculos que establece con estos objetos y que son parte de su familia . Así, cuando Planta se muere, nos cuenta cómo la besa y se despide de ella antes de que su madre la tire por el inodoro: "Ma touches Plant's stalk and . I kiss a few leaves of Plant and flush them, then another few and flush again, then the stalk in bits . 'Good-bye Plant', I whisper. Maybe in the sea she'll stick all back together again and grow up to Heaven" (126). Mientras viven en este habitáculo, Jack crea estos fuertes vínculos con el fin de liberar su ansiedad y miedos inconscientes a la vez que nos lleva hacia una narrativa de recuperación. Jack representa la premisa de los afectos de Tomkins porque es capaz de transformar en dulzura e ingenio sentimientos de culpa insoportables - por no poder ayudar a su madre cuando ésta es violada: "It's my fault for not being dead . I have to Save Ma . ... It's my fault, I made him mad. He can't stand when I start screaming, I haven't done it in years. He wants to punish us" (Donoghue, 2010: 99, 181) .

Si bien puede argumentarse que los niños habitualmente disponen de una mayor afinidad con el mundo de los objetos y son más proclives a jugar con ellos e imaginar, hay que matizar que Jack solo desarrolla estos vínculos afectivos mientras está preso en la habitación. Una vez que Jack y Ma son liberados, con la inestimable ayuda de Alfombra en la que Jack se hace el muerto y Old Nick la usa como envoltorio para deshacerse de él - y salen al exterior, Jack no reconoce a los objetos y ve cómo en el hospital donde pasan unos días, "there's a toilet that's not Toilet and a sink that's twice as big of Sink" (213) y a menudo se pregunta "if Spider is still in Room, if her web is getting bigger and bigger" (344) or if "Rub misses us" (241) . A diferencia de Ma, quien carece de la fuerza necesaria para conectarse a otros afectos, Jack nos muestra cómo la creación de vínculos afectivos con los objetos así como una deslumbrante imaginación son herramientas de resiliencia para alcanzar un cierto bienestar .

\section{CONCLUSIÓN}

La imaginación, si bien nos hace un poco más libres, sirve de puente entre el saber y el no saber, entre lo conocido y lo desconocido, entre la voz que representa al otro dentro de uno mismo y la que retiene la memoria de un suceso traumático. El vínculo que tanto Mala como Jack establecen con otras formas imaginativas de realidad nos ofrece la posibilidad, como críticos lectores, de responder afectivamente a sus traumas a través de prácticas más éticas y justas . Cuando el diálogo no es posible y éste es reemplazado por el silencio - en el caso de Mala su silencio obedece a un dolor negado y a una vergüenza inconfesable y en Jack el silencio representa su alienación absoluta del mundo real - la literatura puede encontrar formas de narrativizarlo para lograr la curación . La literatura, en su afán de imaginar al otro, en su tarea de construir realidades alternativas y de re- 
construir las propias, es un potente antídoto contra la violencia y el odio . Así, además de ser una herramienta estética, la literatura permite narrativizar y visibilizar estos tipos de violencia insidiosa a través de constelaciones reveladoras, entendiendo "constelación" en su sentido estricto acuñado por Walter Benjamin: "una combinación de visiones históricosociales, literarias y políticas que tienen la capacidad de sorprender y de transmitir un sentido emocional transformador" (1968: 90). Es mi convicción que para lograr esta reparación individual y colectiva, debemos realizar un esfuerzo colaborativo que emane tanto de un compromiso personal como de una responsabilidad grupal . Asimismo, generar afectos inducidos de forma positiva y esperanzadora puede forjar nuevos discursos literarios, estéticos y políticos que no nieguen la agencia y la dignidad de las minorías identitarias .

\section{REFERENCIAS BIBLIOGRÁFICAS}

AhMed, S . 2004 . The Cultural Politics of Emotion. London \& New York: Routledge. ARendt, H . 1969 . On Violence. London \& New York: Harcourt Inc.

BenjaMin, W . 1968 . Illuminations. Essays and Reflections. New York: Schocken Books.

BRoWnMilleR, S . 1975 . Against Our Will: Men, Women and Rape. New York: The Ballantine Publishing Group .

Budden, A . 2009 . "The Role of Shame in Post-Traumatic Stress Disorder: A Proposal for a Socio-Emotional Model for DSM-V'. Social Science and Medicine, 69: 1032-39.

CaRuth, C . 1996 . Unclaimed Experience: Trauma, Narrative, and History . Baltimore \& London: The Johns Hopkins UP.

CuoMo, C . 1998 . Feminism and Ecological Communities: An Ethics of Flourishing . London \& New York: Routledge.

Cvetkovick, A . 2003 . An Archive of Feelings: Trauma, Sexuality, and Lesbian Public Cultures. Durham \& London: Duke UP.

CyRulnik, B . 2011 . Resilience: How your Inner Strength Can Set You Free from the Past. New York: Penguin.

Diagnostic and Statistical Manual of Mental Health Disorders (DSM V). 2013. Washington D .C .: American Psychiatric Association .

Donoughue, E . 2010 . Room. Basingstoke \& Oxford: Picador.

Eng, D. \& kazanjian, D . 2003 . (eds .) . Loss . The Politics of Mourning. Berkeley \& Los Angeles . University of California P .

FelMan, S. \& laub, d . 1992 . Testimony: Crises of Witnessing in Literature Psychoanalysis and History. New York: Routledge. 
Freud, S 1957 (1917) . "Mourning and Melancholia" . The Standard Edition of the Complete Psychological Works of Sigmund Freud, Vol. XIV, trad. y ed. James Strachey . London: Hogarth Press .

Henke, S. a . 1998 . Shattered Subjects: Trauma and Testimony in Women's LifeWriting, London: MacMillan .

HeRMan, J. 1 . 1992 . Trauma and Recovery: The Aftermath of Violence - From Domestic Abuse to Political Terror. New York: Basic Books.

LacapRa, D . 2001 . Writing History, Writing Trauma. Baltimore \& London: Johns Hopkins University Press .

Lee, R. \& WheeleR, G . 1996 . The Voice of Shame: Silence and Connection in Psychotherapy. Massachusetts: Gestalt Press .

Lévinas, E. 1998 . Entre-Nous: On Thinking-of-the-Other . Trad . Michael B . Smith and Barbara Harshav. New York: Columbia UP.

LuckhuRst, R . 2008 . The Trauma Question. London \& New York: Routledge.

Mootoo, S . 1996 . Cereus Blooms at Night . London: Granta Books .

Munt, S . 2008 . Queer Attachments: The Cultural Politics of Shame . Hampshire: Ashgate .

PajaczkoWska, C. \& WaRd, I . (eds .) . 2008 . Shame and Sexuality: Psychoanalysis and Visual Culture. London \& New York: Routledge.

Rushdie, S . 1983 . Shame . London: Picador .

Russell, D . 1999. The Secret Trauma: Incest in the Lives of Girls and Women. New York: Basic Books.

SedgWick, E.k. \& FRank, A . 1995 . (eds .). Shame and Its Sisters: A Silvan Tomkins Reader. Durham \& London: Duke UP.

Sontag, S . 2003 . Discurso al Premio Príncipe de Asturias a las Letras . http://www . fpa .es/es/2003-fatema-mernissi-susan-sontag .html?texto=discurso . Accessed 15 Julio 2014.

Tal, K . 1995 . Worlds of Hurt: Reading the Literatures of Trauma . Cambridge: Cambridge UP .

ToMkins, S .1992 [1962] . Affect Imagery Consciousness. 4 vols. New York: Springer.

WateRs, S . 2006 . The Night Watch . London: Virago Press .

Whitehead, A . 2004 . Trauma Fiction . Edinburgh: Edinburgh UP . 